\title{
What is the 'color' of atomic nuclei?
}

\begin{abstract}
A. Schiller, J. A. Becker, L. A. Bernstein, P. E. Garrett, T. S. Hill, D. P. McNabb, W. Younes, E. Tavukcu, A. Voinov, M. Guttormsen, J. Rekstad, S. Siem, R. C. Haight, R. O. Nelson
\end{abstract}

\section{March 18, 2002}

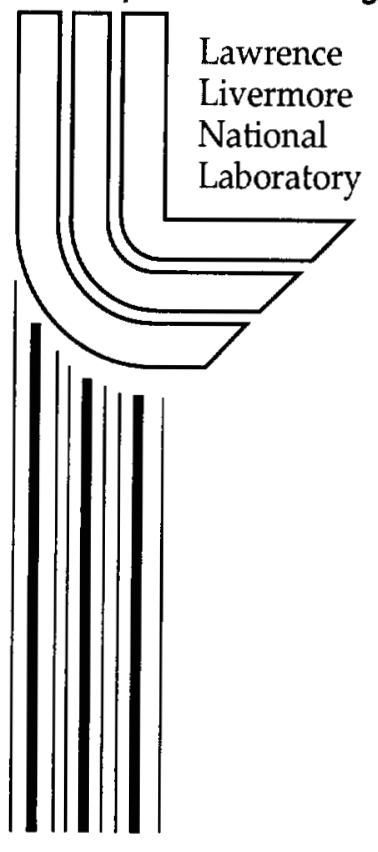


(cross sections) benefit from knowledge of the color of specific nuclei-at least when electromagnetic quanta are participants in the nuclear reaction under study.

\section{Electric and Magnetic Dipoles}

Different kinds of electromagnetic radiation can be observed in atomic nuclei. The simplest ones are electric- and magnetic-dipole radiation. An electric dipole is a simple configuration of a positive and a negative charge, whereas a magnetic dipole is a loop current (Fig. 1). Electromagnetic radiation is produced when the dipole moments begin to oscillate. The difference between electric- and magnetic-dipole radiation is very subtle. The electric dipole viewed in a mirror in Fig. 1 (lower panel) looks as though the positive and negative charges have been interchanged. Thus, the electric-dipole moment $(\vec{d})$ changes direction in the mirror image, and we say that the electric dipole has 'negative parity'. The loop current viewed through a mirror is indistinguishable from the original loop current. Therefore, the magnetic-dipole moment $(\vec{m})$ does not change direction in the mirror image, and we say that the magnetic dipole has 'positive parity'.

In atomic nuclei, an electric dipole occurs when protons and neutrons become separated. The protons in this case are then more positively charged than the average nuclear matter, and, as shown in Fig. 1, they take the place of the positive charge. The neutrons, on the other hand, are more negatively charged than the average nuclear matter, and they take the place of the negative charge. The neutrons and protons will oscillate against each other at a certain frequency and therefore give rise to a huge resonance in the absorption spectrum of electromagnetic radiation. This resonance is located at the oscillation frequency of the protons against the neutrons and is called giant electric-dipole resonance (GEDR) (Fig. 2).

Magnetic dipoles can be realized as (1) the circular motion of a charged particle (proton) orbiting around the center of the nucleus (i.e., orbital magnetic moment) or as (2) the spinning of a particle around itself (i.e., spin magnetic moment). The orbital magnetic moment can be understood in terms of classical mechanics, whereas the spin magnetic moment is a pure quantum effect. In any case, the circular orbital motion on the one hand or the spinning around itself on the other hand takes the place of the loop current in Fig. 1. The spin magnetic moment will start oscillating because the spinning axis is flipped in the nucleus with a certain frequency $\nu_{1}$ due to the influence of the spin-orbit term of the nucleon-nucleon force. As a result, we get the spin flip or giant magnetic-dipole resonance (GMDR) as a second resonance in the absorption spectrum of electromagnetic radiation (Fig. 3). The GMDR is generally weaker (by $\sim 2$ orders of magnitude) than the GEDR, and the resonance frequency of the GMDR is lower (by $\sim 30 \%$ ) than that of the GEDR. The orbital magnetic resonance is discussed in the Fig. 4 caption. 


\section{Experiment}

In a previous experiment, we measured the total $\gamma$-ray absorption spectrum ${ }^{1}$ by the nucleus ${ }^{172} \mathrm{Yb}$ at the Oslo Cyclotron Laboratory in Norway [1]. This absorption spectrum can be described by the sum of the GEDR and GMDR and a third, unknown resonance, which is even weaker and centered at lower frequencies than the GMDR. Our group [2] speculates whether this small resonance is that of the orbital magnetic moment (also called the scissors mode), whereas another group [3] speculates about the possibility of a weak electricdipole resonance at these low frequencies--called the pygmy resonance. These two competing speculations are depicted in Fig. 4.

To test these hypotheses, we excited the ${ }^{172} \mathrm{Yb}$ nucleus by thermal-neutron capture on ${ }^{171} \mathrm{Yb}$ using FP14 at the Lujan Center at LANSCE. This excitation mechanism has the advantage that the energy and parity of the initial state are well determined. We then recorded the spectrum of $\gamma$-ray cascades populating a certain low-lying level in ${ }^{172} \mathrm{Yb}$ by only two $\gamma$-ray transitions. The chosen lowlying level has a parity that is opposite to that of the initial level. This means that the de-excitation by two $\gamma$-ray transitions has to involve one electric- and one magnetic-dipole transition. The sum of energies of both $\gamma$-ray transitions obviously has to match the energy difference between the initial state and the low-lying level, whereby we identify such special decay processes. The two $\gamma$ ray transitions also have to be observed within a short coincidence window of a few $10^{-9}$ seconds. The intensity and the spectral distribution of the two $\gamma$-ray transitions will tell (by means of a difficult-to-explain calculation) whether the third resonance structure at low frequencies is of electric- or orbital magneticdipole character (Fig. 5).

\section{Conclusion}

Preliminary results already show that we collected enough statistics in this experiment to yield a meaningful spectral distribution of two-step $\gamma$-ray transitions. Analysis of the data continues. We hope to soon be able to fill Fig. 5 with data points, favoring either the hypothesis of an electric pygmy resonance (red line) or orbital magnetic scissors mode (blue line).

\section{Acknowledgments}

This work has benefited from the use of the Los Alamos Neutron Science Center at the Los Alamos National Laboratory. This facility is funded by the U.S. Department of Energy under Contract W-7405-ENG-36. Part of this work was performed under the auspices of the U.S. Department of Energy by the University of California, Lawrence Livermore National Laboratory under Contract

\footnotetext{
${ }^{1}$ The actual measured quantity is the total radiative strength function which is proportional to the absorption spectrum.
} 
W-7405-ENG-48, and Los Alamos National Laboratory under Contract W-7405ENG-36. Financial support from the Norwegian Research Council (NFR) is gratefully acknowledged. One of us (A.V.) acknowledges support from a NATO Science Fellowship under project number $150027 / 432$.

\section{References}

[1] A. Voinov et al., $\gamma$-ray strength function and pygmy resonance in rare earth nuclei, Physical Review C 63, 044313 (2001).

[2] A. Schiller et al., Observation of the scissors mode in the quasicontinuum, Los Alamos preprint: http://xxx.lanl.gov/abs/nucl-ex/0011018.

[3] P. Van Isacker et al., Effect of the neutron skin on collective states of nuclei, Physical Review C 45, R13 (1992). 
(a)
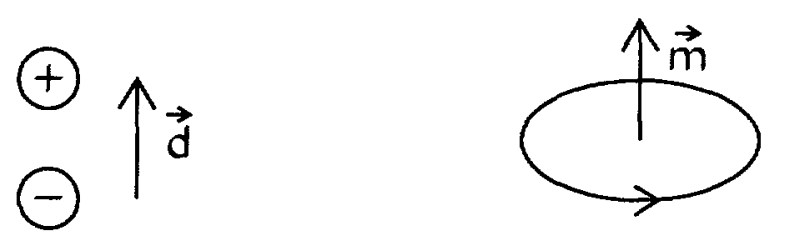

(b)

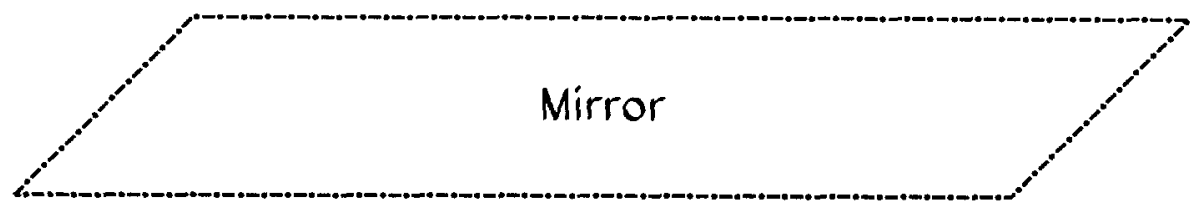

(c)
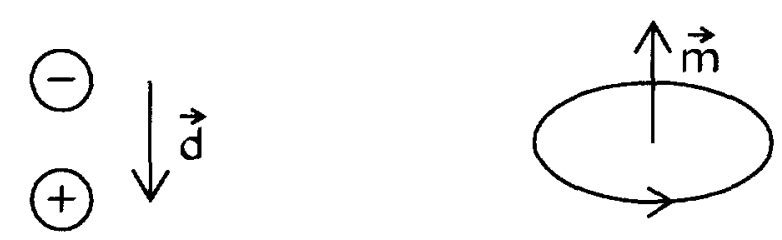

(d)

I

Figure 1: (a) The electric dipole moment $(\vec{d})$ points from the negative charge to the positive charge. (b) The magnetic dipole moment $(\vec{m})$ is perpendicular to the loop current $(I)$. The direction of the magnetic dipole moment is defined such that the loop current circulates counter-clockwise around the magnetic dipole moment. Seen through a mirror, (c) the electric dipole moment changes direction, whereas (d) the magnetic dipole moment does not. 
(a)

(b)
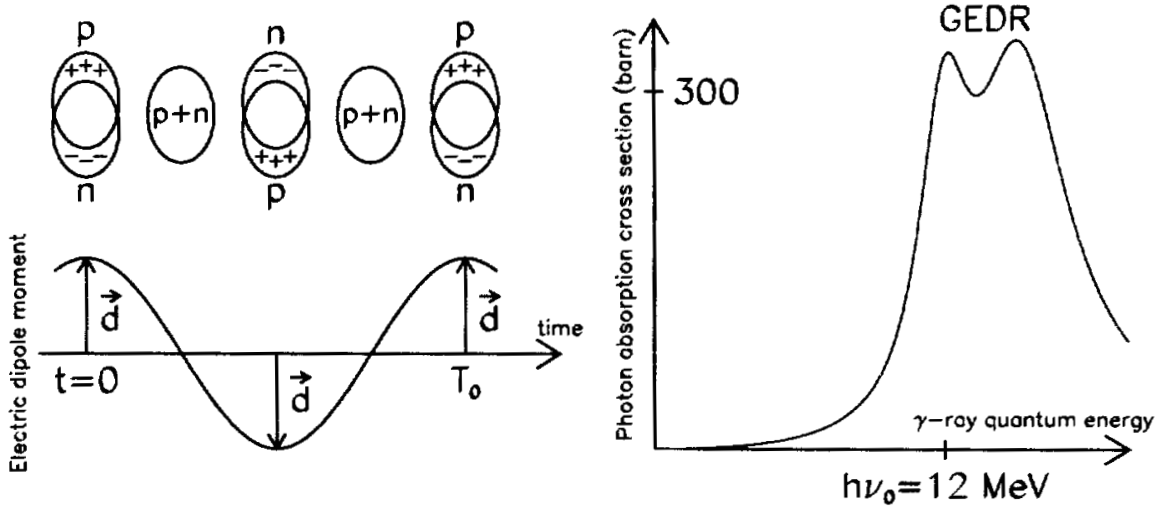

Figure 2: (a) The oscillation of protons versus neutrons in the nucleus and of the connected electric dipole moment $(\vec{d})$ with an oscillation frequency $\nu_{0}=1 / T_{0}$. (b) Resulting absorption spectrum with an electric-dipole resonance (GEDR) at the frequency $\nu_{0}$. The numerical value is for the ${ }^{172} \mathrm{Yb}$ nucleus. The GEDR is double-peaked because the ${ }^{172} \mathrm{Yb}$ nucleus is deformed. 
(a)

(b)
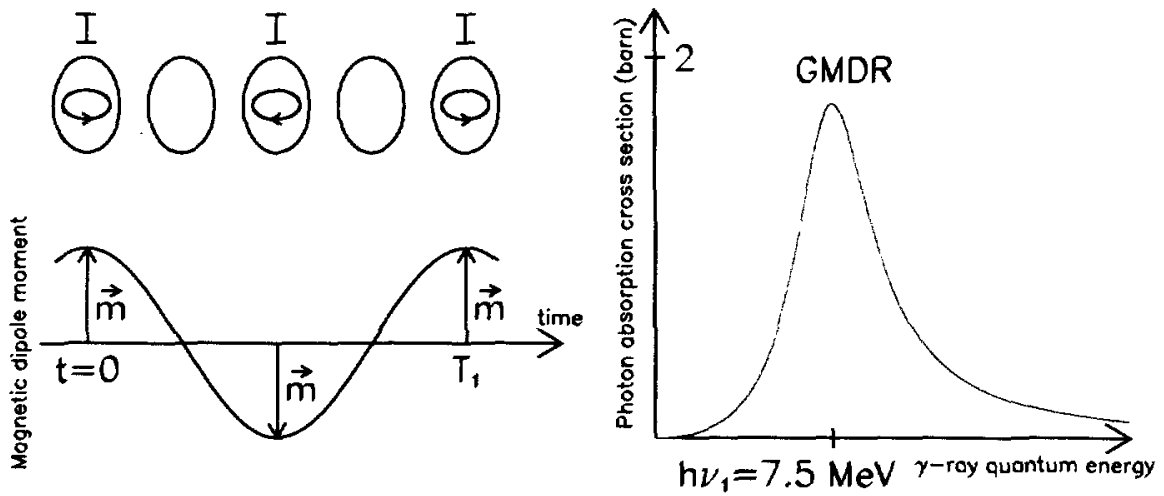

Figure 3: (a) Oscillation of the spin magnetic-dipole moment ( $\vec{m}$, also represented by the loop current $(I)$ ) with respect to time. This oscillation is due to the flipping of the spinning axis with a frequency $\nu_{1}=1 / T_{1}$. (b) Resulting absorption spectrum showing a magnetic-dipole resonance (GMDR) at the frequency $\nu_{1}$. The numerical value is for the ${ }^{172} \mathrm{Yb}$ nucleus. 


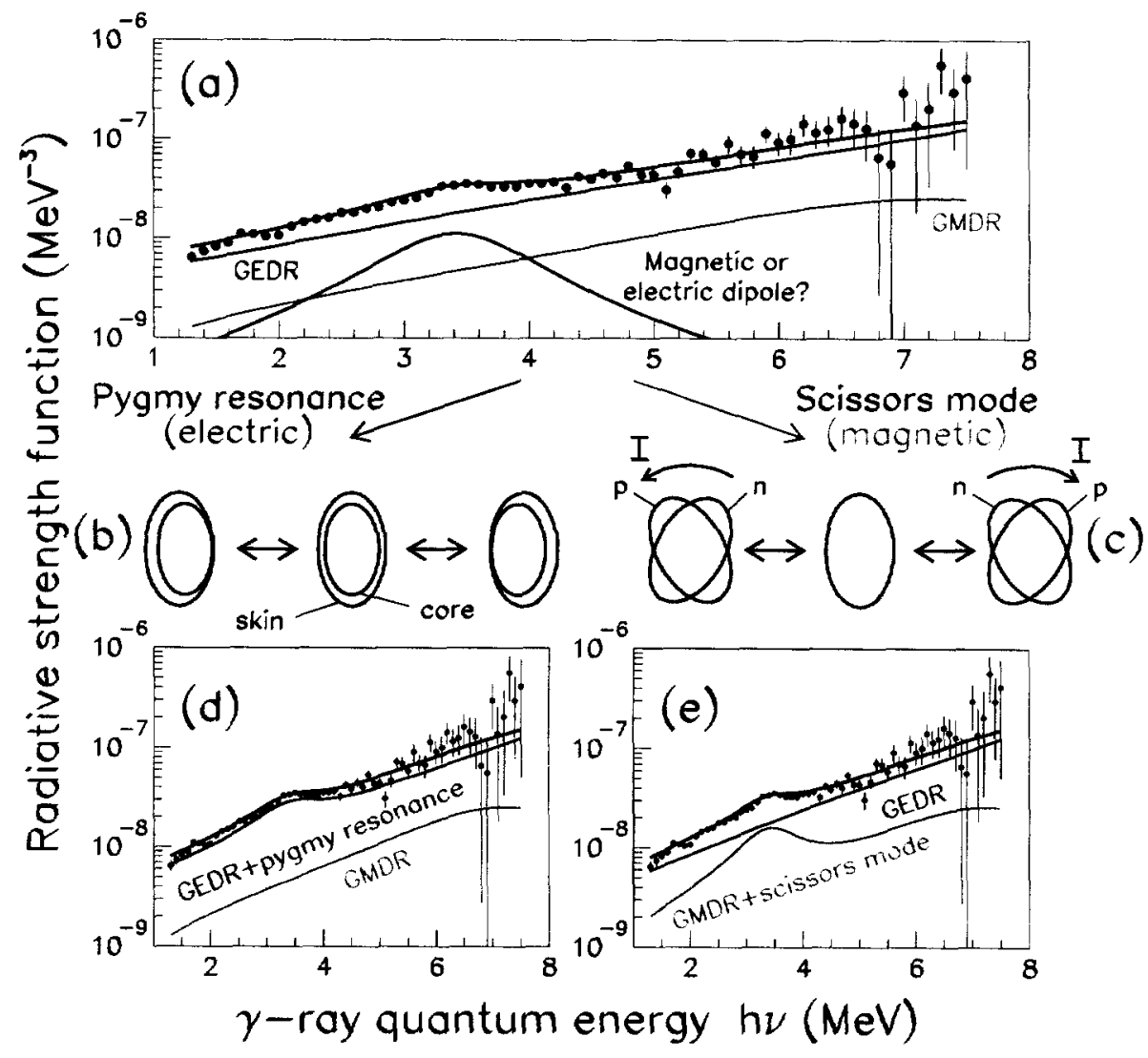

Figure 4: (a) Total radiative strength function of ${ }^{172} \mathrm{Yb}$ (data points from [1]). This strength function can be described as an incoherent sum of the GEDR (red line), the GMDR (blue line) and a third resonance (green line) at lower energy with unknown multipolarity. If the resonance were of the electric-dipole character (pygmy resonance), it would correspond to a vibration of the neutron skin relative to a core of protons and neutrons (b). If the resonance were of the orbital magnetic-dipole character (scissors mode), it would correspond to a counter-wise angular vibration of the protons and neutrons (c) much like the movement of scissors. In any case, this low-energy resonance has to be added (d) either to the already known GEDR it (e) or GMDR to give the total electric or magnetic radiative strength function. 


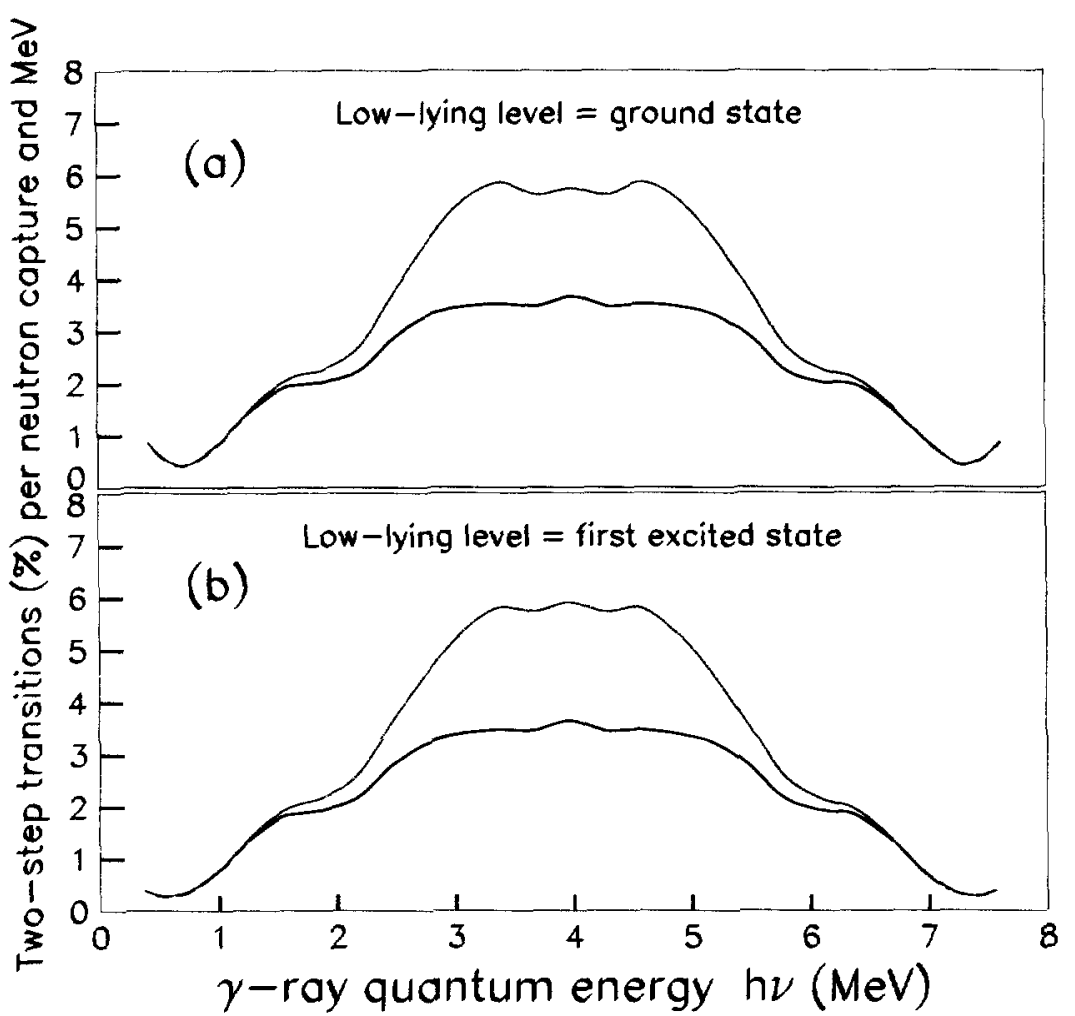

Figure 5: $(\mathrm{a}+\mathrm{b})$ Anticipated spectral distribution of the two $\gamma$-ray transitions after neutron capture. If the unknown resonance is an electric-dipole resonance (pygmy resonance) we expect our data points to ly on the red lines. If it is a magnetic-dipole resonance (scissors mode) we expect our measurement follow the blue lines. The spectral distribution must be symmetric because the energies of both $\gamma$-ray transitions have to add up to the energy difference between the initial state and the chosen low-lying state in the ${ }^{172} \mathrm{Yb}$ nucleus. 